\title{
Carrier effective masses in symmetrically strained (GaIn)As/Ga(PAs) multiple-quantum-well structures
}

\author{
M. Volk, S. Lutgen, ${ }^{*}$ T. Marschner, W. Stolz, and E. O. Göbel ${ }^{\dagger}$ \\ Materials Science Center and Department of Physics, Philipps University, D-35032 Marburg, Germany
}

P. C. M. Christianen and J. C. Maan

High Field Magnet Laboratory and Research Institute for Materials, University of Nijmegen, NL-6525 ED Nijmegen, The Netherlands

(Received 12 June 1995)

\begin{abstract}
The carrier effective masses in symmetrically strained (GaIn)As/Ga(PAs) multiple-quantum-well heterostructures have been determined as a function of the incorporated compressive strain in the (GaIn)As quantum-well layer by applying magneto-optical studies. The precise structural parameters, i.e., individual layer thicknesses, strain values, and crystalline perfection, have been determined independently by high-resolution x-ray diffraction and transmission electron microscopy. By analyzing both the allowed and forbidden optical transitions as a function of the magnetic field, detected by polarization-dependent magnetophotoluminescence excitation spectroscopy, the exciton binding energy as well as the effective in-plane electron and heavy-hole masses have been determined quantitatively as a function of strain. The theoretically predicted significant decrease of the in-plane heavy-hole mass with increasing strain has been observed. The obtained results are discussed and compared with the contradictory results reported in the literature.
\end{abstract}

\section{INTRODUCTION}

The unique possibility in designing the valencesubband structure in strained III/V-semiconductor heterostructures as a function of the incorporated strain has led to new developments with respect to both fundamental physics as well as applications in particular for optoelectronic devices. ${ }^{1-3}$ For compressively strained quantum-well heterostructures, a significant decrease of the in-plane mass of the topmost valence subband with increasing strain has been theoretically predicted. ${ }^{4}$ Most of the studies have been concentrating on the (GaIn)As/GaAs materials system grown epitaxially on the GaAs substrate. Although considerable experimental efforts have been conducted in recent years, the strain dependence of the in-plane valence-band mass has not been clarified unambiguously yet.

The first experimental results were obtained by magnetotransport [Shubnikov-de Haas $(\mathrm{SdH})$ ] investigations of multiple $p$-modulation-doped (GaIn)As/GaAs strainedlayer structures by Schirber and co-workers. ${ }^{5,6}$ In these studies, a constant in-plane valence-band mass of $m_{h}^{*}=0.15$ independent of the incorporated compressive strain is reported. It is important to note, however, that these structures were deposited on a partly relaxed (GaIn)As buffer layer. Therefore, both the constituent (GaIn)As and GaAs layers of the strained-layer superlattice structure are strained. ${ }^{6}$ In addition, it is assumed that the strain-relaxed buffer layers lead to the formation of a great number of dislocations in the epitaxial layer structure. Contrary to these results, SdH studies of $p$ modulation-doped (GaIn)As/GaAs have been reported by Jaffe et al. $^{7}$ where a decreasing hole mass with increasing strain has been observed. Magnetophoto- luminescence (magneto-PL) investigations on a $n$-type modulation-doped (GaIn)As/GaAs/(AlGa)As strained single quantum-well heterostructure (SQWH) yielded a conduction-band effective mass of $m_{e}^{*}=0.07$ and a valence-band mass of $m_{h}^{*}=0.14$ for an In concentration in the well of $x_{\text {In }}=0.15$, assuming parabolic dispersion relations around the zone center. ${ }^{8}$ These authors used impurity-induced forbidden optical transitions, i.e., transitions between conduction- and valence-band Landau levels of different quantum number $N$, in order to extract the electron and hole masses independently. In an extension of these investigations, these authors determined the nonparabolicity of the valence subbands. ${ }^{9}$ An almost constant zone-center mass of about $m_{h}^{*}=0.09$ independent of strain for $0.15<x_{\text {In }}<0.25$ with slightly changing nonparabolicity factors has been found. In the more recent work, the well-width dependence of the exciton binding energy for a fixed value of strain has been investigated using photoluminescence excitation spectroscopy ${ }^{10}$ (PLE) as well as magneto-PL. ${ }^{11,12}$ From a comparison of the determined exciton binding energy and a variational calculation of this quantity, Moore et al. ${ }^{10}$ deduced the in-plane hole mass of $m_{h}^{*}=0.21$ for a $x_{\text {In }}=0.15$ for well widths below $5 \mathrm{~nm}$. From a combination of optically detected cyclotron resonance, yielding the electron mass, and magneto-PL studies using magnetic fields up to $7 \mathrm{~T}$, Mitchell et al. ${ }^{11}$ concluded an increasing hole mass with decreasing well width for (GaIn)As/GaAs QWH having an In concentration in the well layers of $x_{\text {In }}=0.18$. Identical values for the hole mass and a similar dependence as a function of well width have been reported by Pulsford et al. ${ }^{12}$ however, for a $x_{\text {In }}=0.09$ in the (GaIn)As well layer. This again suggests that there exists no straindependent hole mass in this material system. 
In order to clarify this obviously contradictory experimental situation, we present results of a detailed investigation of the magneto-optical properties of symmetrically strained (GaIn)As/Ga(PAs) multiple QWH (MWQH) as a function of the incorporated compressive strain in the (GaIn)As quantum-well layer for constant well width. The precise structural parameters (individual layer thicknesses, strain values) of these strained-layer structures have been determined independently by highresolution $\mathrm{x}$-ray-diffraction (XRD) analysis ${ }^{13}$ and transmission electron microscopy (TEM), establishing the high crystalline perfection of the samples under investigation here. The optical transitions in these strained quantum-well heterostructures have been determined for magnetic fields up to $20 \mathrm{~T}$ using polarization-dependent PLE spectroscopy. These transitions have been analyzed by solving Schrödinger's equation for a two-dimensional exciton in a magnetic field. ${ }^{14}$ The binding energy and the reduced effective mass of the exciton are determined. In addition to the strong allowed optical transitions, also weaker forbidden transitions, corresponding to $p$ - and $d$ type exciton transitions, are observed. The detailed analysis of these forbidden transitions allows for the independent evaluation of the electron and hole masses as a function of strain. This detailed study reveals quantitatively the significant decrease of the in-plane hole mass with increasing strain for a constant well width in symmetrically strained (GaIn)As/Ga(PAs) MQWH.

This paper is organized as follows. The experimental conditions for epitaxial-layer growth and sample characterization as well as the experimental setup for the magneto-optical studies are described in Sec. II. In Sec. III, first the structural and optical characterization establishing the high crystalline perfection of the deposited strained MQWH are presented. The main part of this section is concerned with the description of the experimental polarization-dependent PLE spectra and the detailed analysis of the allowed as well as forbidden optical transitions. This evaluation leads to a quantitative description of the exciton binding energy and, in particular, of the effective electron and hole mass as a function of strain incorporated in the (GaIn)As quantum-well layer. The main conclusions of the presented investigations are summarized in Sec. IV.

\section{EXPERIMENT}

The epitaxial growth of the (GaIn)As/Ga(PAs) symmetrically strained-layer sequence (SLS) structures has been performed by metal-organic vapor-phase epitaxy (MOVPE) in commercial equipment (Aix 200, Aixtron Corp.) at a reactor pressure of 100 mbar and a substrate temperature of $650^{\circ} \mathrm{C}$. The layer sequence for the series of samples consists of 50 periods of a (GaIn)As/GaAs/Ga(PAs)/GaAs building block. The thin GaAs intermediate layers in between the ternary (GaIn)As and Ga(PAs) layers have been deposited in order to avoid the possible formation of strained quaternary (GaIn)(PAs) interface layers. The total strained-layer sequence has been grown on a buffer layer consisting of an (AlGa)As layer and an (AlGa)As/GaAs short-period su- perlattice layer to improve the growth surface morphology. Details of the optimization of these symmetrically SLS structures as well as the evaluation of the straindependent atomic incorporation efficiencies during MOVPE growth have been published elsewhere. ${ }^{13,15}$

The deposited strained-layer structures have been characterized by XRD using a triple crystal diffraction setup $^{16-18}$ (Cu $K_{\alpha 1}$ radiation). In addition to the structural characterization, detailed luminescence studies have been performed in a standard luminescence setup. ${ }^{19}$ The excitation source was an Ar-ion pumped Ti:Sapphire laser system. The samples were mounted in a continuous flow, variable temperature cryostate. The luminescence signal was dispersed by a 1-m single-pass grating monochromator and detected by a liquid-nitrogen cooled $\mathrm{Ge}$ detector using the standard lock-in technique.

The magneto-optical studies have been performed in a Bitter magnet system yielding magnetic fields up to $20 \mathrm{~T}$. The sample was mounted in a liquid $\mathrm{He}$ bath cryostate inside the bore of the magnet system and excited using an Ar-ion pumped Ti:Sapphire layer system. The excitation light was circular polarized by using an achromatic $\lambda / 4$ retarder plate. The luminescence light from the sample was dispersed by a $0.64-\mathrm{m}$ single-pass grating monochromator and detected by a liquid-nitrogen cooled Ge detector using lock-in technique.

\section{RESULTS AND DISCUSSIONS}

\section{A. Structural and optical characterization of symmetrically strained (GaIn)As/Ga(PAs) multiple-quantum-well heterostructures}

In this section the structural and optical properties of the deposited symmetrically strained (GaIn)As/Ga(PAs) MQWH are summarized. Particular emphasis is directed to the exact determination of individual layer thicknesses and strain. The detailed knowledge of these parameters is of key importance in interpreting the results of the exciton binding energies as well as carrier effective masses as a function of strain as determined by magneto-optical investigations. The high-resolution XRD pattern of three samples out of a series of strained MQWH are depicted in Fig. 1. The XRD intensities of these samples are shown on a semilogarithmic scale around the 400 reflection of GaAs. For clarity, the pattern has been shifted on the intensity scale. Besides the reflection of the GaAs substrate and the (AlGa)As buffer layer at a diffraction angle of $0^{\prime \prime}$ and $-200^{\prime \prime}$, respectively, the sharp XRD pattern of the 50-period strained MQWH is clearly detected. For the sample with the lowest In concentration (top), the main reflection of the MQWH is detected in between the substrate and the buffer-layer peaks, while for the other samples the main MQWH reflection coincides with the substrate reflection. The average total strain in these samples is below $\pm 1 \times 10^{-3}$ indicating the almost perfect strain balancing in these highly strained MQWH samples. The XRD linewidths of the MQWH main reflection as well as of the satellite reflections in the range of 21-28' (FWHM) are only slightly above the theoretical values of about $20^{\prime \prime}$. Details of the XRD eval- 


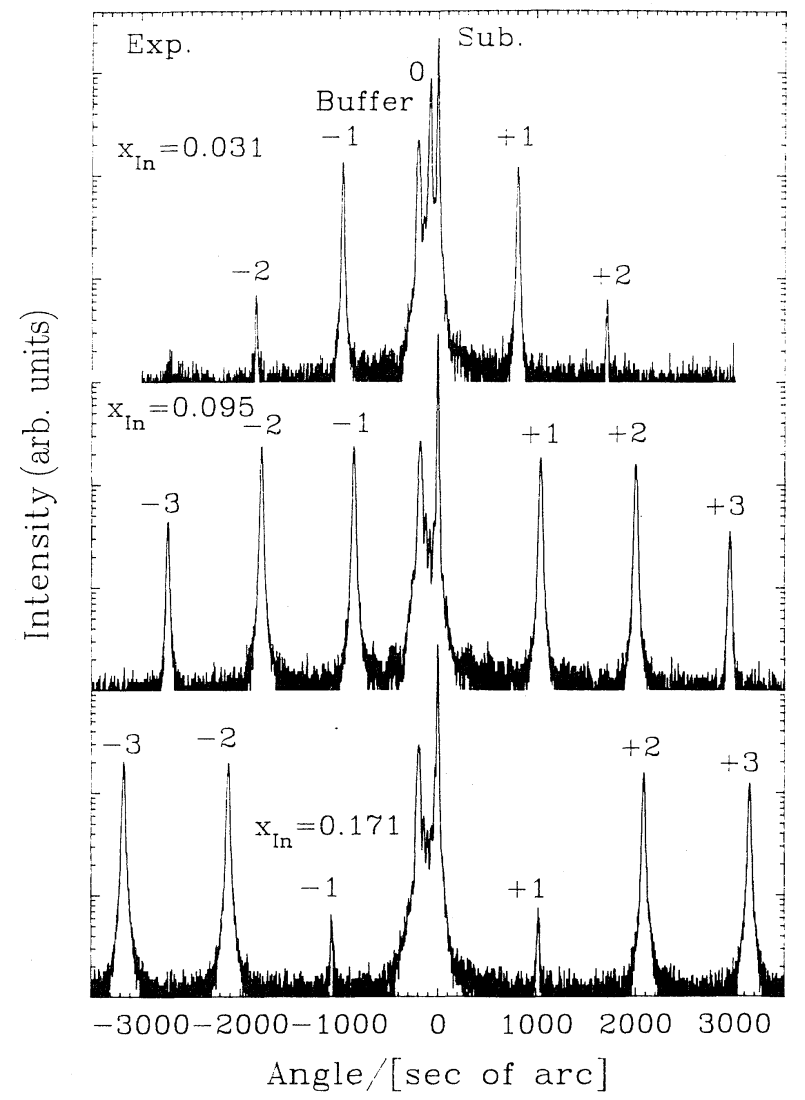

FIG. 1. High-resolution $x$-ray diffraction pattern around the 400 reflection of $\mathrm{GaAs}\left(\mathrm{Cu} K_{\alpha 1}\right.$ radiation) on a semilogarithmic plot of 50-period symmetrically strained (GaIn)As/Ga(PAs) MQWH for different values of the incorporated strain in the individual ternary layers as indicated (Ref. 13). The XRD pattern is shifted on the intensity scale for clarity.

uation of the series of samples can be found elsewhere. ${ }^{13}$ From this evaluation, which is based on a theoretical description of the experimental XRD pattern using the dynamical $x$-ray diffraction theory, the precise values of the individual layer thicknesses and strain of all constituent layers can be extracted. The data for the samples selected for magneto-optical investigations are summarized in Table $I$.

For part of the samples, TEM investigations have been performed. As an example, the TEM micrograph of one of the structures is shown in Fig. 2. Because of the exact strain balancing in the symmetrically strained
(GaIn)As/Ga(PAs) layer sequence, defect-free MQWH with a lattice mismatch of up to $2.4 \%$ in the individual layers can be realized. The interface abruptness is comparable to standard unstrained (AlGa)As/GaAs heterostructures. The individual layer thicknesses as determined from the XRD data analysis are identical to the values extracted from the TEM investigations. The XRD and TEM investigations underline the structural perfection of the deposited symmetrically strained (GaIn)As/Ga(PAs) MQWH, having a total strained-layer thickness of $1 \mu \mathrm{m}$.

In addition to these structural characterization methods, detailed PL investigations have been performed using both PL and PLE spectroscopy, in order to clarify the crystalline perfection of the deposited symmetrically strained (GaIn)As/Ga(PAs) MQWH. The PL (dotted lines) and PLE (full line) spectra of the four samples selected for the magneto-optical studies at low temperature and no applied magnetic field are summarized in Fig. 3. For clarity, the individual spectra are shifted on the intensity scale. Sharp excitonic resonances associated with the different subband transitions as indicated are observed. For the lowest $e_{1}-\mathrm{hh}_{1}$ exciton resonances (where hh denotes heavy hole), a clear separation from the continuum transitions is observed. Linewidths of the excitonic PL and PLE resonance amount to values of 2-3 and 4-5 meV (FWHM), respectively. The Stokes shift between the emission and absorption process is about 2 meV. In more detail the optical properties with no applied magnetic field are discussed elsewhere. ${ }^{19}$ The results of the structural as well as the optical characterization techniques establish the high crystalline perfection of these highly strained MQWH.

\section{B. Magneto-optical investigations of strained (GaIn)As/Ga(PAs) MQWH}

In this section the experimental photoluminescence excitation spectra of the different samples are presented for varying strengths of the applied magnetic field perpendicular to the MQWH layer sequence. The observed characteristic optical transitions are described using the model of a two-dimensional exciton in a magnetic field. From the analysis of the characteristic energy shifts of the allowed as well as forbidden transitions, the exciton binding energy as well as the reduced effective mass and the individual electron and hole masses are determined as a function of the compressive strain in the (GaIn)As quantum-well layer.

TABLE I. Structural parameters of the (GaIn)As/Ga(PAs) symmetrical SLS as determined by x-ray diffraction analysis. The individual layer thicknesses are determined within an error bar of $0.5 \mathrm{~nm}$, the individual compositions within a relative error of about $5 \%$ (Ref. 13).

\begin{tabular}{cccccccc}
\hline \hline No. & $x_{\mathrm{In}}$ & $\begin{array}{c}d_{(\mathrm{GaIn}) \mathrm{As}} \\
(\mathrm{nm})\end{array}$ & $\begin{array}{c}d_{\mathrm{GaAs} 1} \\
(\mathrm{~nm})\end{array}$ & $y_{P}$ & $\begin{array}{c}d_{\mathrm{Ga} \text { (PAs })} \\
(\mathrm{nm})\end{array}$ & $\begin{array}{c}d_{\mathrm{GaAs} 2} \\
(\mathrm{~nm})\end{array}$ & $\begin{array}{c}D_{\mathrm{MQWH}} \\
(\mathrm{nm})\end{array}$ \\
\hline 1 & 0.052 & 9.2 & 1.7 & 0.100 & 8.8 & 1.8 & 21.5 \\
2 & 0.079 & 9.1 & 1.2 & 0.206 & 8.6 & 1.5 & 20.3 \\
3 & 0.124 & 8.5 & 1.2 & 0.272 & 8.2 & 1.6 & 19.5 \\
4 & 0.156 & 7.8 & 1.3 & 0.334 & 7.6 & 1.4 & 18.1 \\
\hline \hline
\end{tabular}




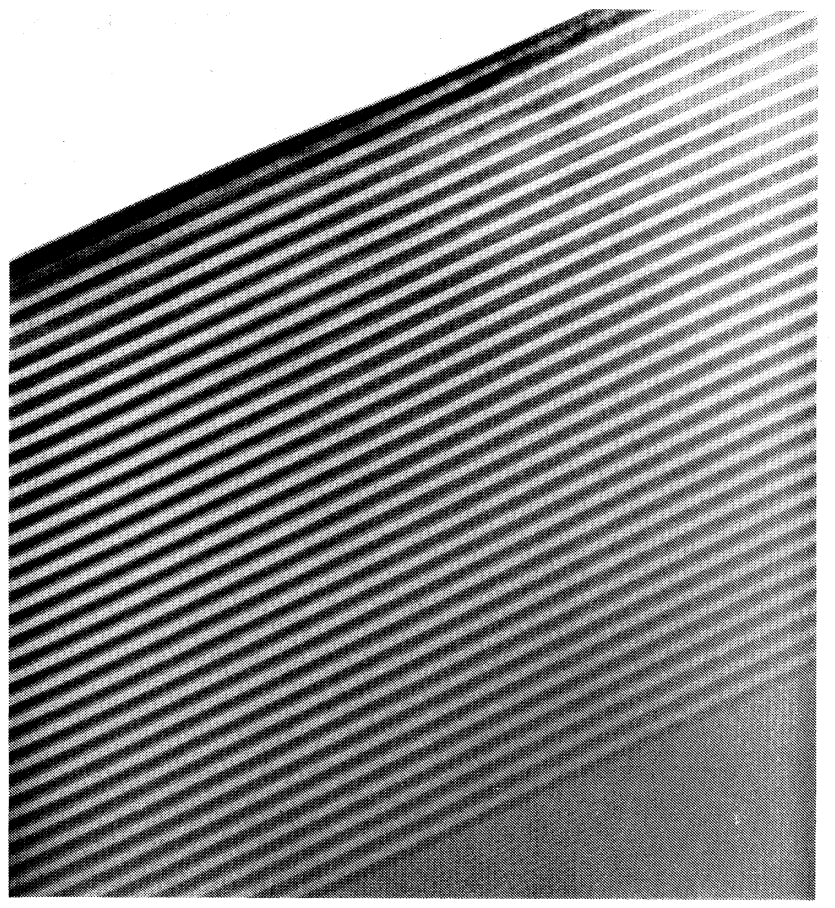

FIG. 2. Transmission electron microscopy (TEM) micrograph of the layer sequence of a symmetrically strained (GaIn)As/Ga(PAs) MQWH with an In concentration of $x_{\text {In }}=0.192$.

The experimentally obtained excitation spectra for two of the samples with the magnetic field as a measurement parameter are summarized in Fig. 4. Similar sets of PLE spectra are recorded for the other samples. The full and dashed lines represent, respectively, the data obtained from left and right circular polarized light excitation. For clarity, the individual spectra have been shifted on the intensity scale. The evolution of the Landau-level fan structure is clearly detected for all samples already at low magnetic fields in the range of $1-2 T$, pointing again to the high crystalline perfection of these samples. In addition to the strong peak structures, resulting from allowed optical transitions, weaker peak structures, indicated by arrows in Fig. 4, are observed in particular for high magnetic fields.

The experimentally determined Landau-level transitions are analyzed by solving Schrödinger's equation for a two-dimensional exciton in a magnetic field ${ }^{14}$

$$
\begin{gathered}
{\left[-\partial^{2 / \partial} \rho^{2}-1 / \rho \partial / \partial \rho+m^{2} / \rho^{2}-2 / \rho+(\gamma \rho)^{2} / 4\right] R_{n, m}(\rho)} \\
=E_{n, m} R_{n, m}(\rho) \\
E_{\mathrm{tot}, n, m}=E_{n, m}-\left(m_{e}-m_{h}\right) /\left(m_{e}+m_{h}\right)^{*} \gamma m
\end{gathered}
$$

In these equations energies are expressed in units of the Rydberg $R_{y}=\mu e^{4} / 2\left(4 \pi \epsilon \hbar^{2}\right)^{2}$, lengths in units of Bohr radius $a_{B}=4 \pi \epsilon \hbar^{2} / \mu e^{2}$, and $\gamma$ is given by the ratio of $\gamma=\hbar \omega_{c} / 2 R_{y}$. Two approaches have been applied to solve Eqs. (1) and (2). The first one is the direct numerical solution of Schrödinger's equation. As the exciton is not strictly two-dimensional in the present MQWH, the

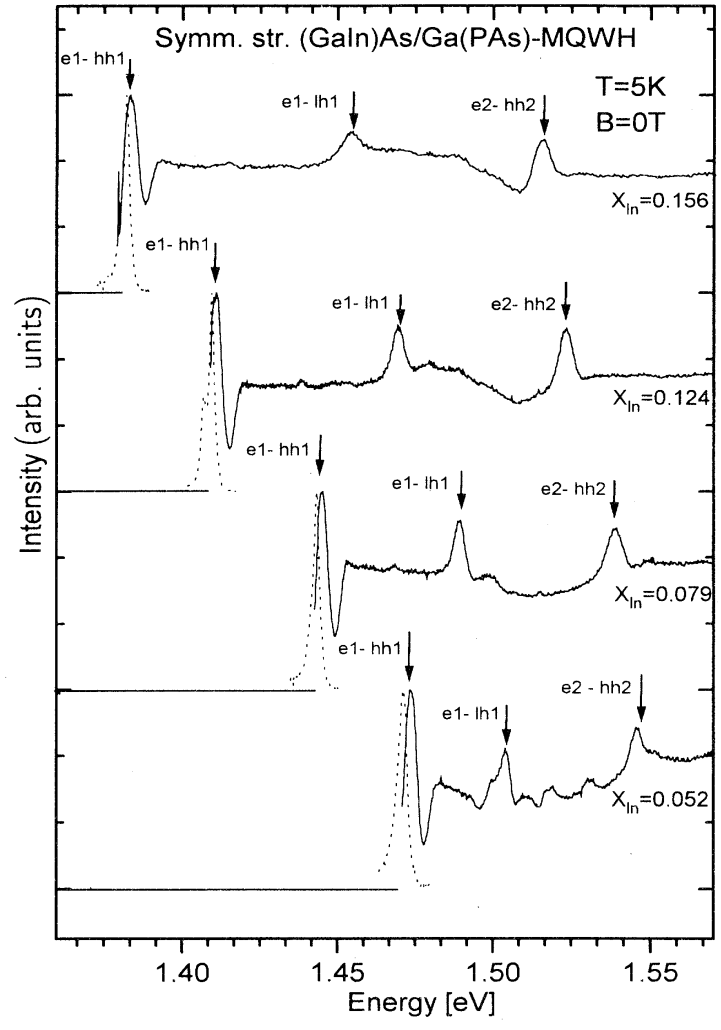

FIG. 3. Photoluminescence (PL) (dashed) and photoluminescence excitation (PLE) (full line) spectra of symmetrically strained (GaIn)As/Ga(PAs) MQWH for different values of the In concentration as indicated at a temperature of $5 \mathrm{~K}$ with no applied magnetic field. The PL and PLE spectra for different samples are shifted on the intensity scale for clarity. Experimentally observed excitonic resonances in the PLE spectra are labeled according to the respective subband transition.

exciton binding energy is taken as a parameter in this evaluation as in the literature for similar magneto-optical experiments. ${ }^{20-22}$ This scaling of the exciton binding energy is similar to the introduction of a scaling factor for the exciton dimensionality. ${ }^{23}$ The second approach is an extension of the analytic two-point Padé approximation of the hydrogenic energy levels in two dimensions at arbitrary magnetic fields, as introduced by MacDonald and Ritchie. ${ }^{24}$ This analytic solution has been extended by taking the finite hole mass of the exciton explicitly into account. Both methods yield the same result; however, the use of the analytic approximation leads to a drastic reduction in computation time.

The solutions of the Eqs. (1) and (2) depend strongly on the quantum number $m$. The value of $m=0$ corresponds to $s$-type solutions in terms of the exciton model and to $\Delta N=0$ in terms of the Landau-level transitions, i.e., transitions between Landau levels of electrons and holes with equal Landau-level quantum number $N$. In principle $k$ conservation implies that only $m=0$ transitions are allowed; therefore, only Eq. (1) has to be considered, which only depends on the binding energy and the reduced effective mass of the exciton. This analysis is the standard procedure for obtaining these two quantities from 
the experimental data, as has been performed for various material systems in the literature. ${ }^{22,25-27}$ This method, however, does not directly lead to the electron and hole effective mass. Further assumptions or the knowledge of one of the two quantities, in general, is necessary in order to determine the other.

The forbidden transitions with $m= \pm 1, m= \pm 2$ correspond to $p$ - and $d$-type solutions in the exciton picture, or
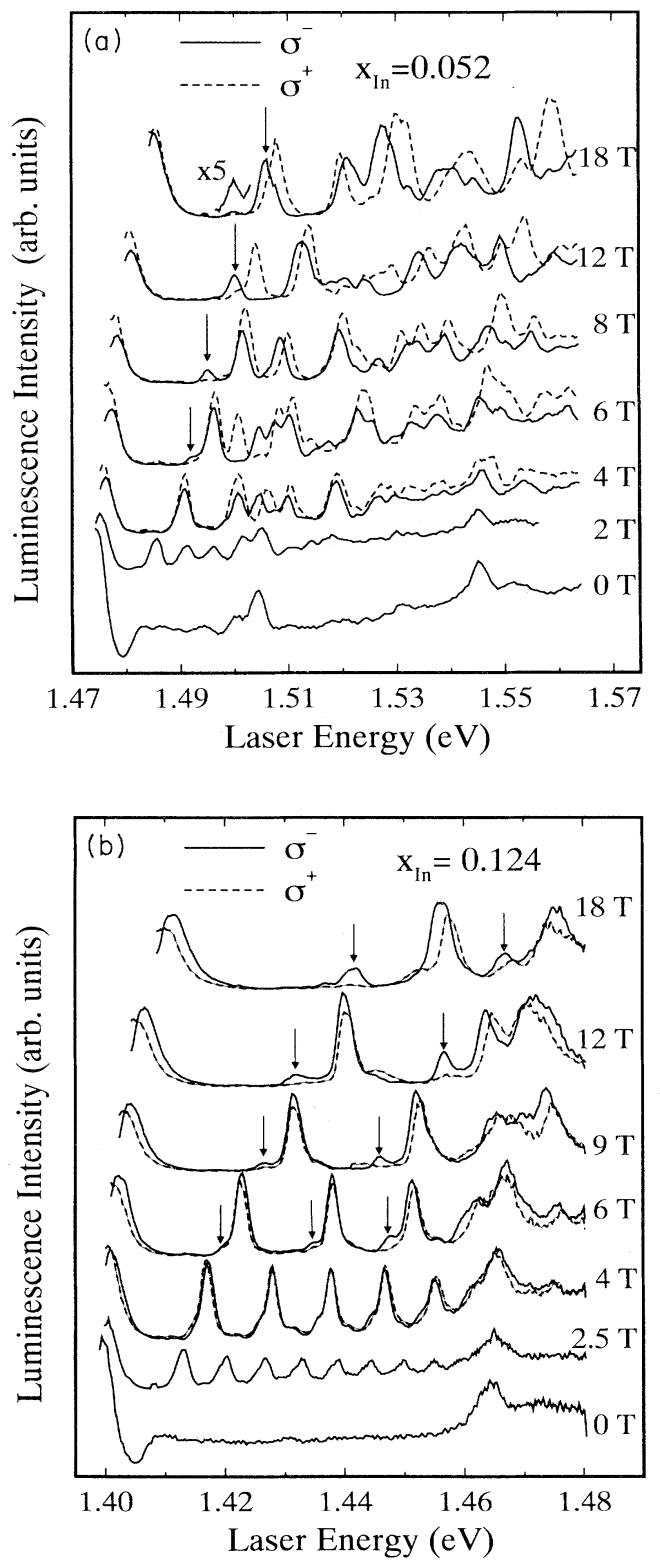

FIG. 4. Photoluminescence excitation spectra of symmetrically strained (GaIn)As/Ga(PAs) MQWH as a function of the applied magnetic field perpendicular to the MQWH structure for different values of the In concentration in the compressive strained (GaIn)As quantum well (a) $x_{\text {In }}=0.052$ and (b) $x_{\text {In }}=0.124$. The full (dashed) line represents PLE spectra recorded using left (right) circular polarized light excitation. to transitions between electron and hole Landau levels of different Landau quantum numbers $N .{ }^{14}$ As possible mechanisms for the observation of these transitions, scattering mechanisms (impurities, ${ }^{6}$ interface roughness or alloy disorder) as well as the presence of electric fields ${ }^{27}$ are discussed. Because of the high crystalline perfection of the series of samples under investigation here, structural inhomogeneities should not be the major reason for breaking of the selection rules in the magnetooptical studies. Presumably, the "forbidden" transitions become partly allowed due to the presence of a surface charge depletion field in these samples. The residual $n$ type background doping level of around $1 \times 10^{15} \mathrm{~cm}^{-3}$ for the (GaIn)As layers leads to a surface depletion layer thickness on the order of $1 \mu \mathrm{m}$, which is equal to the total strained MQWH layer thickness. Therefore, we assume a perpendicular electric field on the order of $5 \mathrm{kV} / \mathrm{cm}$ to be present in these samples. Vina et al. ${ }^{27}$ showed that with the application of an electric field in this range, forbidden transitions become apparent in the spectra of unstrained quantum-well structures, because of symmetry breaking.

The Landau-level fan charts of two of the samples under investigation here for left circular polarized light excitation are summarized in Fig. 5. The symbol size represents the strength of the experimental detected optical transitions. The lines describe the theoretical dependence of the model of the two-dimensional exciton in a magnetic field. Full lines describe optical transition energies of $m=0$ ( $s$-type) excitonic transitions, while $p$-type and $d$-type transitions are represented by the dotted and dashed lines, respectively. The parameters entering the theoretical model are the exciton binding energy and the electron and hole effective masses. For the forbidden transitions with $m \neq 0$, the transition energy depends not only on the reduced mass but also on the ratio of electron and hole mass [see also Eq. (2)]. Therefore, by an analysis, which takes also these forbidden transitions into account, it is possible to determine independently the values for the effective electron and hole mass. To the best of our knowledge this is the first paper to use the Landau-level fan chart of the forbidden transitions to extract the electron and hole masses directly from the experimentally determined PLE transition energies. A similar method using the occurrence of forbidden transitions in the emission process detected by PL spectroscopy, however, is only applicable to doped heterostructures. ${ }^{6}$

In order to determine the different carrier effective masses, the magnetic-field dependence of the $2 p$ and $3 d$ transitions has been studied particularly, because these transitions are most clearly separated from all other transitions in the complicated Landau-level fan charts (Fig. 5). It is important to note that the $3 d$ transition has a polarization dependence as predicted by theory by Bauer and Ando. ${ }^{28}$ However, the $2 p$ transition is weakly observed experimentally for both polarizations in contrast to the theoretical description. ${ }^{28}$ Also in the case of the unstrained QWH the $2 p$ - as well as the $2 p+$ transitions are detected experimentally for both polarizations. ${ }^{27}$ Further studies are underway to clarify this specific polarization dependence of the different $2 p$ transitions. 


\section{Discussion of carrier effective masses in strained (GaIn)As/Ga(PAs) MQWH}

The values for the exciton binding energy and the carrier effective masses, which have been obtained as presented in the previous section are summarized in Table II and will be discussed in more detail as a function of strain in this section.

The exciton binding energy determined from the magneto-optical investigations agrees within the error bars with results obtained from the transient four-wavemixing (FWM) experiments reported earlier on the same set of samples. ${ }^{29}$ These values are significantly larger than the data obtained from the line-shape analysis of the PLE spectra recorded without applied magnetic field. ${ }^{19}$ In this study, lower exciton binding energies in the range of 5-6 meV have been extracted, using the absorption model of an ideal exciton in two dimensions. ${ }^{30-32}$ Further investigations are underway in particular with respect to possible changes in the relaxation processes of excited carriers near the band edge in order to clarify the discrepancy between the excitation spectra and the twodimensional absorption model. As we would like to point out, however, the correct values for the exciton binding energy in the symmetrically strained (GaIn)As/Ga(PAs) MQWH are directly determined by the FWM (Ref. 29) and the magneto-optical studies reported here. For small values of the In concentration, an increase in exciton binding energy from the GaAs bulk value is observed, while for higher values a saturation of the exciton binding energy at a value of $10 \mathrm{meV}( \pm 1.5 \mathrm{meV})$ is found. It is important to note that these exciton binding energies and the effective electron and hole masses, as determined here independently by the additional analysis for the forbidden transitions at high magnetic fields, are consistent with one another.

The determined values of the carrier effective masses are summarized in Fig. 6, where the different masses are plotted as a function of the incorporated compressive strain determined by the In concentration in the (GaIn)As quantum wells. The electron mass in these MQWH is almost independent of the strain value in the quantum-well layer with a value of $m_{e}^{*}=0.067$. With increasing In concentration, the confinement of the carriers in the quantum wells is increasing. This is caused in the symmetrically strained (GaIn)As/Ga(PAs) MQWH by a simultaneous decrease in (GaIn)As band edge and an increase in $\mathrm{Ga}$ (PAs) barrier height with increasing In concentration. Therefore, the reduction in the electron mass
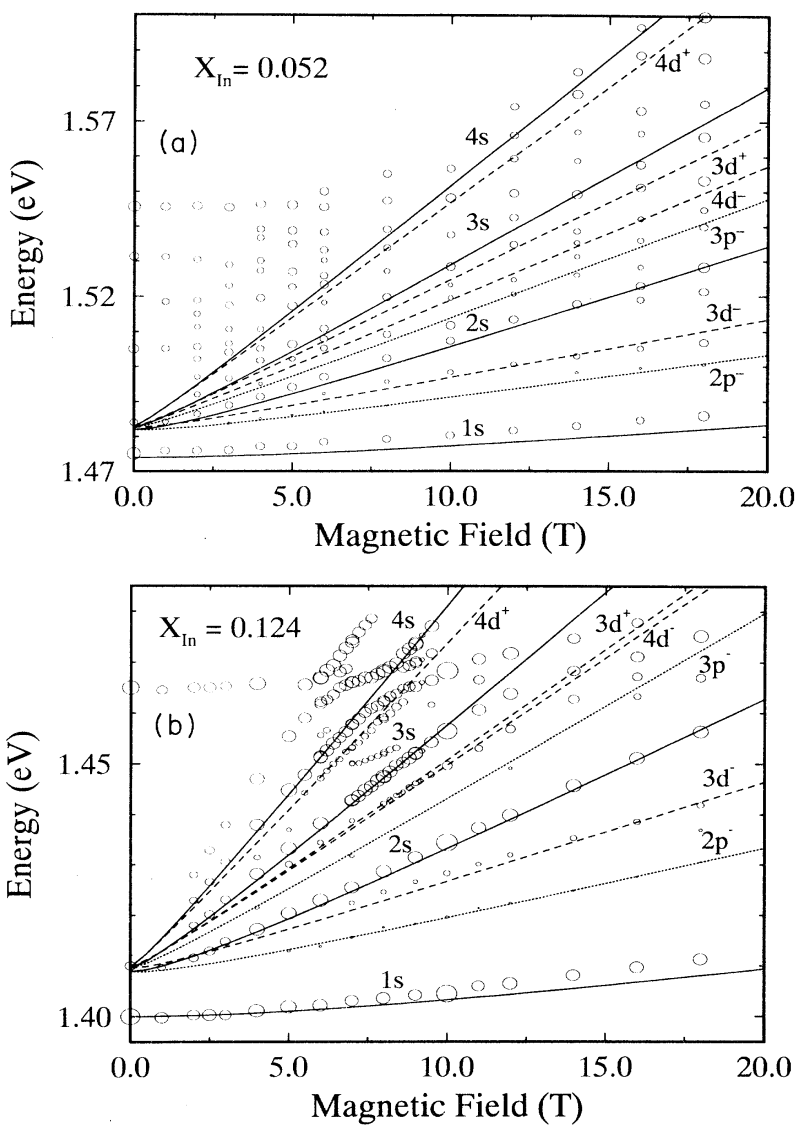

FIG. 5. Landau-level fan chart of symmetrically strained (GaIn)As/Ga(PAs) MQWH for different values of the In concentration in the compressive strained (GaIn)As quantum well (a) $x_{\text {In }}=0.052$ and (b) $x_{\text {In }}=0.124$. Experimentally observed optical transitions are plotted as circles. The respective transition strength is indicated by the symbol size for the various optical transitions. The lines result from the theoretical description using the two-dimensional exciton in a magnetic field model as detailed in the text. The full lines represent allowed $s$-type exciton transitions, while $p$-type and $d$-type exciton transitions are shown by dotted and dashed lines, respectively.

with increasing In concentration is compensated by the increased nonparabolicity of the conduction band due to the stronger confinement in these structures. It is important to note that the electron masses determined here agree well with theoretical calculations taking nonparabolicity corrections into account. ${ }^{33,34}$ For

TABLE II. Exciton binding energies $E_{X}$ and carrier effective masses in units of the free-electron mass $m_{0}$ of the symmetrically strained (GaIn)As/Ga(PAs) MQWH. The error bars of the different quantities as determined from the theoretical analysis are indicated.

\begin{tabular}{ccccccc}
\hline \hline No. & $x_{\mathrm{In}}$ & $\begin{array}{c}\epsilon_{(\mathrm{GaIn}) \mathrm{As}} \\
(\%)\end{array}$ & $\begin{array}{c}E_{X}(\mathrm{meV}) \\
\pm 1.5\end{array}$ & $\begin{array}{c}m_{e}\left(m_{0}\right) \\
\pm 0.001\end{array}$ & $\begin{array}{c}m_{\mathrm{hh}}\left(m_{0}\right) \\
\pm 0.03\end{array}$ & $\begin{array}{c}\mu\left(m_{0}\right) \\
\pm 0.002\end{array}$ \\
\hline 1 & 0.052 & 0.37 & 9.0 & 0.0674 & 0.30 & 0.056 \\
2 & 0.079 & 0.58 & 10.0 & 0.0672 & 0.25 & 0.054 \\
3 & 0.124 & 0.90 & 10.0 & 0.0670 & 0.22 & 0.053 \\
4 & 0.156 & 1.12 & 10.0 & 0.0667 & 0.19 & 0.052 \\
\hline \hline
\end{tabular}




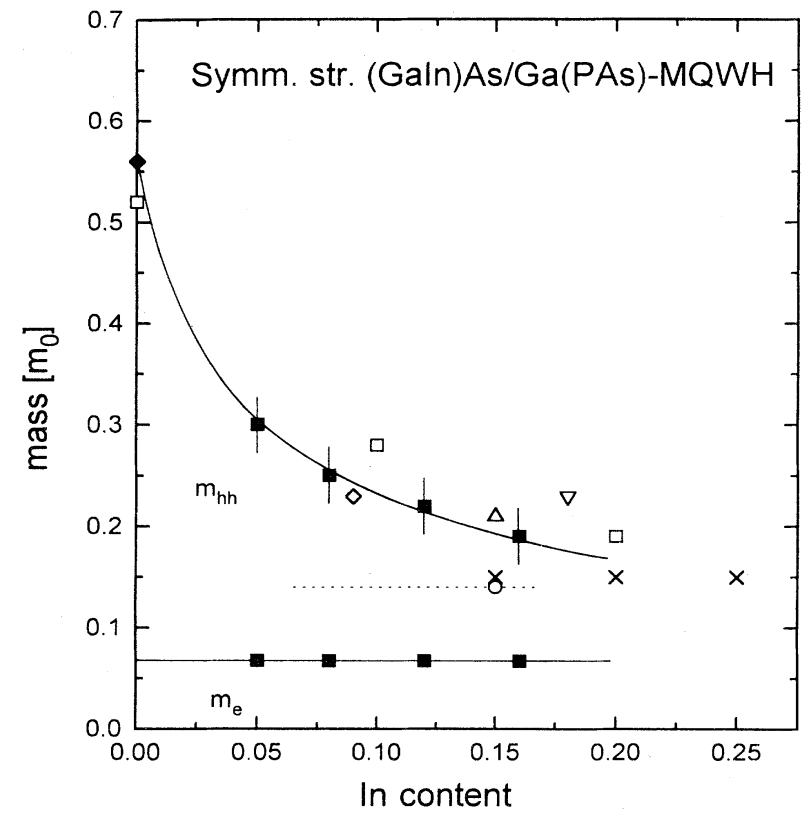

FIG. 6. Carrier effective masses in strained (GaIn)As QWH as a function of the In concentration. The full squares are the data points, obtained in this work on symmetrically strained (GaIn)As/Ga(PAs) MQWH by magneto-PLE spectroscopy. The full line is a guide to the eye. Open symbols represent literature data obtained on(GaIn)As/GaAs MQHW [dotted line (Refs. 5 and 6), squares (Ref. 7), circle (Ref. 8), crosses (Ref. 9), upward triangle (Ref. 10), downward triangle (Ref. 11), open rhombus (Ref. 12), and full rhombus (Ref. 20)].

(GaIn)As/GaAs QWH, a value of the electron mass of $m_{e}=0.07$ for $x_{\mathrm{In}}=0.17$ has been reported. ${ }^{6,9}$ This value is in accordance with the data presented here for the (GaIn)As/Ga(PAs) material system.

As determined from the standard procedure, i.e., evaluating $s$-type exciton transitions as a function of magnetic field, the reduced effective exciton mass is primarily determined by the electron mass in these structures. Therefore, the hole mass can only be determined with a large error bar. Using the analysis of the forbidden transitions, however, this mass can be extracted independently with much higher accuracy. Simulations of the various Landau-level transitions with varying hole masses show that the hole mass can be determined with an accuracy of better than $0.03 m_{0}$. For the effective in-plane heavy-hole mass, a decrease with increasing In concentration in the (GaIn)As quantum well is observed as shown in Fig. 6. It is important to note that it is not possible to describe the magnetic-field dependence of the forbidden transitions using a constant hole mass for these structures. Therefore, the observed decrease in the hole effective mass with increasing In concentration is proven unambiguously. The values for the in-plane heavy-hole mass decrease from 0.6 for an unstrained GaAs/(AlGa)As QWH of similar well width ${ }^{20}$ to a value of 0.19 for a strain value of $1.1 \%$ in the symmetrically strained (GaIn)As/Ga(PAs) MQHW. The literature data of the (GaIn)As/GaAs-material system, if available for a quantum-well width in the range of $8-10 \mathrm{~nm}$ are also included. Good agreement is obtained with part of the literature data for specific In concentrations in the (GaIn)As quantum-well material. As a result of the present study, a consistent dependence of the in-plane heavy-hole valence-subband mass as a function of In concentration is established for this material system, which is very important also from an applied point of view.

In the following, we will briefly estimate whether the experimentally detected changes in the valence-subband structure are primarily caused by the incorporated strain or the confinement in these particular MQWH. The increase in the in-plane mass of the topmost heavy-hole subband results from the mixing of this subband with the lower lying lh (light-hole) valence subband. ${ }^{4,25}$ This mixing can be reduced, and thus, the in-plane heavy-hole mass decreased by an increased energetic separation of the individual valence subbands. This can be achieved on one hand by a stronger confinement, i.e., by a reduction of the well width or an increase in barrier height. This leads to an increase energy splitting of the $\mathrm{hh}$ and $\mathrm{lh}$ valence subbands due to the different quantization masses. A resulting reduction in the in-plane hh mass has been reported by Rogers et al. ${ }^{20}$ for unstrained GaAs/(AlGa)As QWH with decreasing well width and, thus, increasing confinement. On the other hand, the incorporation of biaxial strain leads to a lifting of the $\mathrm{hh}$ and $\mathrm{lh}$ degeneracy in the Brillouin-zone center. ${ }^{35}$ In order to clarify whether the increase in strain or in confinement causes the observed in-plane hh mass reduction with increasing In concentration, the strain-induced energy splitting is calculated and compared to the experimentally observed energy separation of the $e_{1}-\mathrm{hh}_{1}$ and $e_{1}-\mathrm{lh}_{1}$ exciton resonances in the PLE spectra (see also Fig. 3). The hh-lh valence-band splitting is calculated using the relation ${ }^{35}$

$$
\Delta E_{\mathrm{hh}-\mathrm{lh}}=2 b^{*}\left(C_{11}+2 C_{12}\right) / C_{11}^{*} \epsilon,
$$

where the shear stress deformation potential $b$ is taken to $b=1.7 \mathrm{eV}$. The elastic stiffness coefficients are linearly interpolated between the values for the binary GaAs and InAs, ${ }^{36}$ and the in-plane strain $\epsilon$ is taken from Table I as determined by XRD. According to this calculation 80-90\% of the experimentally observed energy splittings are caused by the incorporated compressive strain, while the remaining percentage originates from the quantum confinement splitting. This proves unambiguously that the experimentally observed reduction of the in-plane hh mass is primarily due to the incorporated strain in the investigation (GaIn)As/Ga(PAs) MQWH.

It is important to note that the in-plane hh dispersion is strongly nonparabolic. ${ }^{4,6,25}$ The determined masses here should therefore represent a mass averaged over a certain range of $k$ states near the Brillouin-zone center. This holds true for all the PL and PLE spectroscopy data summarized in Fig. 6. For the transport studies, the determined hole mass represents the value at the Fermi energy, which depends on the actual doping concentration. In the present study, an upper limit estimate of the averaged part of the dispersion curve can be obtained from the magnetic length scale for a field of $20 \mathrm{~T}$, the 
highest value of the magnetic field used here. The magnetic length of $5 \mathrm{~nm}$ yields a corresponding value of $k_{\|}=0.02 \mathrm{~A}^{-1}$. A detailed description also using theoretical subband dispersion calculations in a magnetic field is underway and will be discussed elsewhere.

\section{SUMMARY}

The carrier effective masses in symmetrically strained (GaIn)As/Ga(PAs) multiple-quantum-well heterostructures have been determined as a function of the incorporated compressive strain in the (GaIn)As quantum-well layer using magneto-optical studies. The precise structural parameters, i.e., individual layer thicknesses, strain values, and crystalline perfection have been determined by high-resolution XRD and TEM independent of the optical studies. Due to the almost perfect strain balancing in these 50-period MQWH having a total layer thickness of $1 \mu \mathrm{m}$, high-quality MQWH with sharp XRD reflections and abrupt heterointerfaces have been realized. The energy shift of the observed optical transitions in the PLE as a function of the magnetic field has been analyzed using the solution of Schrödinger's equation for a two-dimensional exciton in a magnetic field. Both strong allowed optical transitions as well as weaker forbidden transitions are experimentally detected. The ob- servation of the symmetry breaking forbidden transitions is presumably caused by the surface charge depletion electric field present in the structures. By analyzing both the allowed and forbidden optical transitions, the exciton binding energy, the reduced mass, and in addition the effective electron and hole masses are precisely determined as a function of strain. The theoretically predicted significant decrease of the in-plane heavy-hole mass with increasing strain has been observed unambiguously. The obtained results are discussed and compared to the contradictory results reported in the literature. We conclude that on the basis of our data, a consistent description of the heavy-hole in-plane for compressive-strained (GaIn)As QWH is obtained.

\section{ACKNOWLEDGMENTS}

Expert technical support by $T$. Ochs during MOVPE growth is gratefully acknowledged. The authors would like to thank Dr. N.Y. Jin-Phillipp and Dr. F. Phillipp (Max-Planck Institute for Metal Research Stuttgart, for performing TEM investigations on part of the samples investigated here). This work has been supported by the Deutsche Forschungsgemeinschaft (DFG, Bonn) and by the Commission of the European Community in the "Large Installations Plan."
${ }^{*}$ Present address: Max-Born-Institut für Nichtlineare Optik und Kurzzeitspektroskopie, D-12489 Berlin, Germany.

†Present address: Physikalisch-Technische Bundesanstalt (PTB), D-38116 Braunschweig, Germany.

${ }^{1}$ A. Adams, Electron. Lett. 22, 249 (1986).

${ }^{2}$ E. Yablonovitch et al., J. Lightwave Technol. LT-4, 504 (1986)

${ }^{3}$ E.g., P. J. A. Thijs, Proceedings of the 13 th International Semiconductor Laser Conference, Takamatsu, 1992 [IEEE Conf. Dig. 1992, 2].

${ }^{4}$ E.g., J. Singh, in Condensed Systems of Low Dimensionality, Vol. 253 of NATO Advanced Study Institute, Series B: Physics, edited by J. L. Beeby (Plenum, New York, 1991), p. 653.

${ }^{5}$ J. E. Schirber, I. J. Fritz, and L. R. Dawson, Appl. Phys. Lett. 46, 187 (1985).

${ }^{6}$ G. C. Osbourn, J. E. Schirber, T. J. Drummond, L. R. Dawson, B. L. Doyle, and I. J. Fritz, Appl. Phys. Lett. 49, 731 (1986).

${ }^{7}$ M. Jaffe, J. E. Oh, J. Pamulapati, J. Singh, and P. Bhattacharya, Appl. Phys. Lett. 54, 2345 (1989).

${ }^{8}$ S. K. Lyo, E. D. Jones, and J. F. Klem, Phys. Rev. Lett. 61, 2265 (1988).

${ }^{9}$ E. D. Jones, S. K. Lyo, I. J. Fritz, J. F. Klem, J. E. Schriber, C. P. Tigges, and T. J. Drummond, Appl. Phys. Lett. 54, 2227 (1989).

${ }^{10}$ K. J. Moore, G. Duggan, K. Woodbridge, and C. Roberts, Phys. Rev. B 41, 1090 (1990).

${ }^{11}$ K. Mitchell, N. Ahmed, M. J. L. S. Haines, S. J. A. Adams, I. R. Agool, M. G. Wright, C. R. Pidgeon, B. C. Cavenett, E. P. O. Reilly, A. Ghiti, W. Batty, and M. T. Emeny, in Proceedings of the 20th International Conference of Physics of Semiconductors, Thessaloniki, 1990, edited by E. M. Anastassakis and J. D. Joannopoulos (World Scientific, Singapore, 1990), Vol. 2, p. 957.

${ }^{12}$ N. J. Pulsford, R. J. Nicholas, R. J. Warburton, G. Duggan,
K. J. Moore, K. Woodbridge, and C. Roberts, Phys. Rev. B 43, 2246 (1991).

${ }^{13}$ S. Lutgen, T. Marschner, W. Stolz, E. O. Göbel, and L. Tapfer, J. Cryst. Growth 152, 1 (1995).

${ }^{14}$ J. C. Maan, in The Physics of Low-Dimensional Semiconductor Structures, edited by P. Butcher et al. (Plenum, New York, 1993), p. 333.

${ }^{15}$ S. Lutgen, T. Marschner, T. F. Albrecht, W. Stolz, E. O. Göbel, and L. Tapfer, in Proceedings of the European Materials Research Society Symposium, Strasbourg, 1992 [Mater. Sci. Eng. B 21, 249 (1993)].

${ }^{16}$ M. Renninger, Acta Crystallogr. 8, 597 (1955).

${ }^{17}$ M. Renninger, Z. Naturforsch. Teil A 16, 1110 (1961).

${ }^{18}$ M. Renninger, Adv. X-Ray Anal. 10, 32 (1967).

${ }^{19}$ S. Lutgen, T. F. Albrecht, T. Marschner, W. Stolz, and E. O. Göbel, in Proceedings of the Sixth International Conference on Modulated Semiconductor Structures, GarmischPartenkirchen, 1993 [Solid-State Electron. 37, 9005 (1994)].

${ }^{20}$ D. C. Rogers, J. Singleton, R. J. Nicholas, C. T. Foxon, and K. Woodbridge, Phys. Rev. B 34, 4002 (1986).

${ }^{21}$ F. Ancilotto, A. Fasolino, and J. C. Maan, Phys. Rev. B 38, 1788 (1988).

${ }^{22}$ L. Vina, L. Munoz, N. Mestres, E. S. Koteles, A. Ghiti, E. P. O'Reilly, D. C. Bertolet, and K. M. Lau, Phys. Rev. B 47, 13926 (1993).

${ }^{23}$ H. Mathieu, P. Lefebvre, and P. Christol, J. Appl. Phys. 72, 300 (1992).

${ }^{24}$ A. H. MacDonald and D. S. Ritchie, Phys. Rev. B 33, 8336 (1986).

${ }^{25}$ J.C. Maan, G. Belle, A. Fasolino, M. Altarelli, and K. Ploog, Phys. Rev. B 30, 2253 (1984).

${ }^{26}$ M. Potemski, L. Vina, G. E. W. Bauer, J. C. Maan, K. Ploog, and G. Weimann, Phys. Rev. B 43, 14707 (1991). 
${ }^{27}$ L. Vina, G. E. W. Bauer, M. Potemski, J. C. Maan, E. E. Mendez, and W. I. Wang, Phys. Rev. B 41, 10767 (1990).

${ }^{28}$ G. E. W. Bauer and T. Ando, Phys. Rev. B 38, 6015 (1988).

${ }^{29}$ M. Koch, M. Volk. T. Meier, J. Feldmann, W. Stolz, P. Thomas, and E. O. Göbel, Superlatt. Microstruct. 15, 329 (1994).

${ }^{30}$ M. Shinada and S. Sugano, J. Phys. Soc. Jpn. 21, 1936 (1966).

${ }^{31}$ H. Iwamura, H. Kobayashi, and H. Okamoto, Jpn. J. Appl. Phys. 23, L795 (1984).

${ }^{32}$ W. Stolz, J. C. Maan, M. Altarelli, and K. Ploog, Phys. Rev. B
36, 4301 (1987).

${ }^{33}$ U. Ekenberg and M. Altarelli, Phys. Rev. B 32, 3712 (1985).

${ }^{34}$ U. Ekenberg, Phys. Rev. B 36, 6152 (1987).

${ }^{35}$ F. H. Pollak and M. Cardona, Phys. Rev. 172, 816 (1968).

${ }^{36}$ Physics of Group IV Elements and III/V Compounds, edited by $O$. Madelung, M. Schulz, and H. Weiss, LandoltBörnstein, New Series, Group III, Vol. 17, Part a (SpringerVerlag, Berlin, 1982), and references therein. 


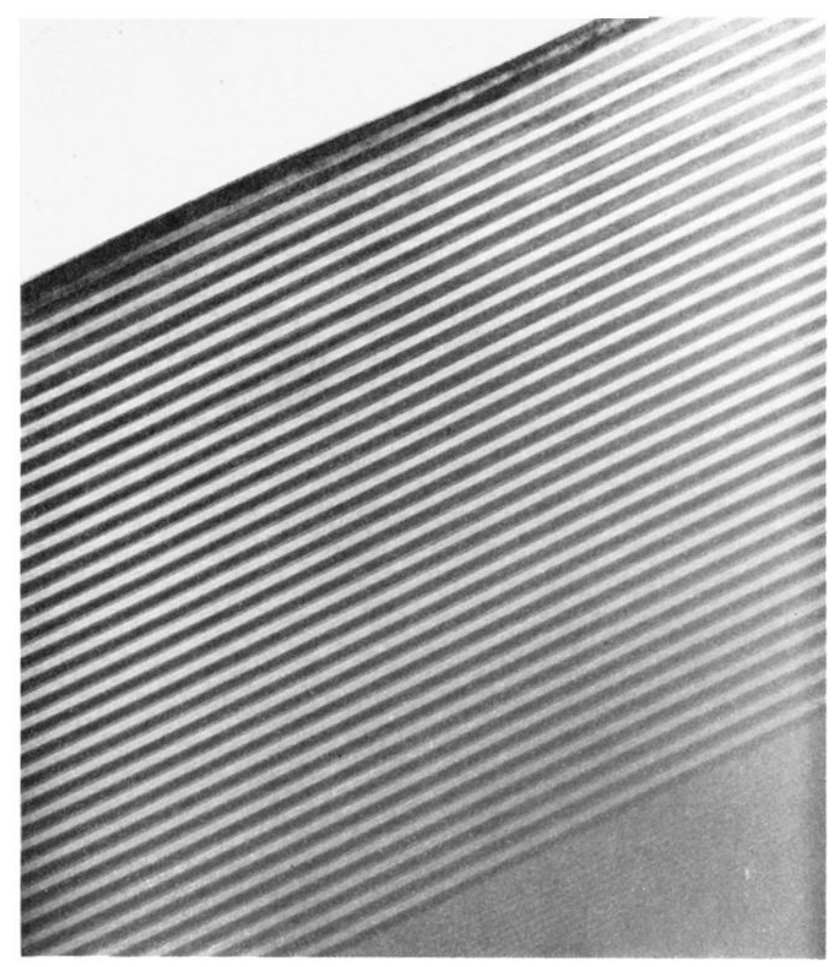

FIG. 2. Transmission electron microscopy (TEM) micrograph of the layer sequence of a symmetrically strained (GaIn)As/Ga(PAs) MQWH with an In concentration of $x_{\text {In }}=0.192$. 